\title{
Potential Health and Environmental Risks of Three-Dimensional Engineered Polymers
}

Marcia de Almeida Monteiro Melo Ferraz, ${ }^{\dagger}$ Heiko H. W. Henning, ${ }^{\ddagger}$ Pedro Ferreira da Costa, ${ }^{\S}, \|$ Jos Malda, ${ }^{\ddagger, \S, \|}$ Séverine Le Gac, ${ }^{\perp}$ Fabrice Bray, ${ }^{\#}$ Majorie B. M. van Duursen, ${ }^{@}$ Jos F. Brouwers, ${ }^{\nabla}$ Chris H. A. van de Lest, ${ }^{\ddagger, \nabla}$ Ingeborg Bertijn, ${ }^{\dagger, \nabla}$ Lisa Kraneburg, ${ }^{\dagger, \nabla}$ Peter L. A. M. Vos, ${ }^{\dagger}$ Tom A. E. Stout, ${ }^{\dagger \dagger}$ and Barend M. Gadella ${ }^{*} \dagger, \nabla_{\infty}$

${ }^{\dagger}$ Department of Farm Animal Health, Faculty of Veterinary Medicine, Utrecht University, Utrecht 3584CM, The Netherlands ${ }^{\ddagger}$ Department of Equine Sciences, Faculty of Veterinary Medicine, Utrecht University, Utrecht 3584CM, The Netherlands

${ }^{\S}$ Department of Orthopedics, Utrecht Medical Center, Utrecht 3584CX, The Netherlands

"Utrecht Biofabrication Facility, Utrecht Medical Center, Utrecht 3584CX, The Netherlands

${ }^{\perp}$ Applied Microfluidics for Bioengineering Research, MESA+ Institute for Nanotechnology and MIRA Institute for Biomedical Technology and Technical Medicine, University of Twente, Enschede 7522 NB, The Netherlands

${ }^{\#}$ Miniaturization for Synthesis, Analysis and Proteomics, USR CNRS 3290, University of Lille, Lille 59650, France

${ }^{\circledR}$ Institute for Risk Assessment Sciences, Division of Toxicology and Pharmacology, Faculty of Veterinary Medicine, Utrecht University, Utrecht 3584CM, The Netherlands

${ }^{\nabla}$ Department of Biochemistry and Cell Biology, Faculty of Veterinary Medicine, Utrecht University, Utrecht 3584CM, The Netherlands

\section{Supporting Information}

ABSTRACT: Polymer engineering, such as in three-dimensional (3D) printing, is rapidly gaining popularity, not only in the scientific and medical fields but also in the community in general. However, little is known about the toxicity of engineered materials. Therefore, we assessed the toxicity of 3Dprinted and molded parts from five different polymers commonly used for prototyping, fabrication of organ-on-a-chip platforms, and medical devices. Toxic effects of PIC100, E-Shell200, E-Shell300, polydimethylsiloxane, and polystyrene (PS) on early bovine embryo development, on the transactivation of estrogen receptors were assessed, and possible polymer-leached components were identified by mass spectrometry. Embryo development beyond the twocell stage was inhibited by PIC100, E-Shell200, and E-Shell300 and correlated to the released amount of diethyl phthalate and polyethylene glycol. Furthermore, all polymers (except PS) induced estrogen receptor transactivation. The released materials from PIC100 inhibited embryo cleavage across a confluent monolayer culture of oviduct epithelial cells and also inhibited oocyte maturation. These findings highlight the need for cautious use of engineered polymers for household 3D printing and bioengineering of culture and medical devices and the need for the safe disposal of used devices and associated waste.

\section{INTRODUCTION}

In addition to the research and biomedical fields, the frequency of use of three-dimensional (3D) printing has increased greatly in industry and for domestic uses, mostly because the cost of 3D printers has dropped dramatically. ${ }^{1}$ Despite the increase in the frequency of use of $3 \mathrm{D}$ engineering in various fields, the effects of the plastics used on the bioengineered culture systems, the users, or the environment have not been widely investigated. ${ }^{1}$ However, 3D-printed parts have been reported to be toxic to zebrafish embryos, ${ }^{1,2}$ to cause allergic and inflammatory responses in mice, ${ }^{3}$ and to be toxic to cancer cells. ${ }^{4}$ Moreover, previous studies have shown that some $3 \mathrm{D}$ printers release toxic particles into the air during printing. ${ }^{5}$ Because of the rapid growth in the scientific and medical applications of $3 \mathrm{D}$ printing, together with an increase in domestic hobby-related use and the large amounts of waste produced, it is important to determine whether bioengineered polymers are toxic, and the degree to which they might be. Toxicological screening of bioengineered materials is also of

Received: November 3, 2017

Revised: December 30, 2017

Accepted: January 5, 2018

Published: January 5, 2018 
great importance before one can decide to safely use such materials for medical and biological applications. ${ }^{2,6-8}$

In this study, we monitored the developmental fate of bovine zygotes over time when they were cultured in media in contact with PIC100, E-Shell200, E-Shell300, polydimethylsiloxane (PDMS), or polystyrene (PS). These plastics are used in $3 \mathrm{D}$ cell culture, medical devices, and industry prototyping applications. It is known that endocrine disruptors are released from plastics, and such components can cause adverse developmental, ${ }^{9}$ reproductive, ${ }^{10,11}$ neurological, ${ }^{12}$ and immune ${ }^{13}$ effects in humans, domestic animals, and wild animals. Therefore, we also investigated possible endocrine disruptor action using an estrogen receptor (ER) transactivation assay and identified leached compounds by mass spectrometry (MS).

\section{MATERIALS AND METHODS}

Chemicals. Unless stated otherwise, all chemicals were obtained from Sigma Chemical Co. (St. Louis, MO) and were of the highest available purity.

Polystyrene (PS) and Polydimethylsiloxane (PDMS) Structures. A two-component kit (Sylgard 184, Dow Corning, Farnell, Maarssen, The Netherlands) consisting of a prepolymer and curing agent was used to fabricate PDMS structures. These two components were mixed at a 10:1 weight ratio. This mixture was degassed and poured onto a silicon wafer, and curing was performed at $60{ }^{\circ} \mathrm{C}$ overnight. The PDMS layer was then released from the silicon wafer and cut into pieces that were $1 \mathrm{~cm}$ in diameter and $0.2 \mathrm{~cm}$ in thickness. For PS, CELLSTAR Petri dishes (Greiner, Bio-One GmbH, Alphen aan de Rijn, The Netherlands) were cut into pieces that were $1 \mathrm{~cm}$ in diameter and $0.2 \mathrm{~cm}$ in thickness.

Production, Prewashing, and Sterilization of 3DPrinted PIC 100, E-Shell200, and E-Shell300 Structures. The prototype design of the structures (discs that were $1 \mathrm{~cm}$ in diameter and $0.2 \mathrm{~cm}$ in thickness) was created using Tinkercad (Autodesk Inc.). Devices were 3D-printed using a photopolymerization process and a Perfactory 3 mini $3 \mathrm{D}$ printer (Envisiontec $\mathrm{GmbH}$, Gladbeck, Germany) at a resolution of 50 $\mu \mathrm{m}$, in various materials: PIC100, E-Shell200, and E-Shell300 (Envisiontec $\mathrm{GmbH}$ ). The safety data sheet for each material can be accessed via https://envisiontec.com/wp-content/ uploads/2017/08/MK-SDS-PIC-series-v.2-USA.pdf, https:// envisiontec.com/wp-content/uploads/2016/09/SDS-E-shell200-V1.5-161028-E.pdf, and https://envisiontec.com/wpcontent/uploads/2017/10/SDS-E-shell-300-V1.5-161028-E.pdf for PIC100, E-Shell200, and E-Shell300, respectively. To limit leakage of compounds from the PIC100 3D-printed structures, the excess resin was removed via a $15 \mathrm{~min}$ immersion in ethanol at room temperature. After being completely air-dried, the structures were immersed three times for $2 \mathrm{~h}$ in isopropanol at room temperature. After being air-dried again, the structures were light-cured using 4000 flashes from an Otoflash G171 instrument (Envisiontec $\mathrm{GmbH}$ ).

All polymer structures were then sterilized by immersion for $1 \mathrm{~h}$ in $70 \%$ ethanol, washed three times for $30 \mathrm{~min}$ in a phosphate-buffered saline (PBS) solution, and then washed for $1 \mathrm{~h}$ in HEPES-buffered Medium 199 supplemented with 100 units $/ \mathrm{mL}$ penicillin and $100 \mu \mathrm{g} / \mathrm{mL}$ streptomycin, at room temperature.

Oocyte Collection, in Vitro Maturation (IVM), and Sperm Preparation for in Vitro Fertilization (IVF). Bovine ovaries were collected from slaughterhouses and prepared as described previously. ${ }^{14}$ The oocytes were allowed to mature in groups of 50 cumulus-oocyte complexes (COCs) in $500 \mu \mathrm{L}$ of maturation medium M199 supplemented with $0.02 \mathrm{IU} / \mathrm{mL}$ follicle-stimulating hormone (Sioux Biochemical Inc., Sioux Center, IA), $0.02 \mathrm{IU} / \mathrm{mL}$ luteinizing hormone (Sioux Biochemical Inc.), $7.71 \mu \mathrm{g} / \mathrm{mL}$ cysteamine, $10 \mathrm{ng} / \mathrm{mL}$ epidermal growth factor in $0.1 \%(\mathrm{w} / \mathrm{v})$ fatty acid-free bovine serum albumin (BSA), 100 units/mL penicillin, and $100 \mu \mathrm{g} /$ $\mathrm{mL}$ streptomycin and incubated in a humidified atmosphere of $5 \% \mathrm{CO}_{2}$ in air for $24 \mathrm{~h}$ at $38.5{ }^{\circ} \mathrm{C}$. Frozen spermatozoa were thawed and prepared as described previously. ${ }^{14}$ Briefly, $250 \mu \mathrm{L}$ straws of cryopreserved spermatozoa were thawed at $37^{\circ} \mathrm{C}$ for $30 \mathrm{~s}$, and the spermatozoa were then washed by centrifugation at $700 \mathrm{~g}$ for $30 \mathrm{~min}$ through a discontinuous Percoll gradient (GE Healthcare) at $27^{\circ} \mathrm{C}$. The supernatant was removed, and the sperm pellet was resuspended in fertilization medium (modified Tyrode's medium supplemented with $25 \mathrm{mM}$ sodium bicarbonate, $22 \mathrm{mM}$ lactate, $1 \mathrm{mM}$ pyruvate, and 6 $\mathrm{mg} / \mathrm{mL}$ fatty acid-free BSA containing 100 units $/ \mathrm{mL}$ penicillin and $100 \mu \mathrm{g} / \mathrm{mL}$ streptomycin). The effect of leaching of PIC100 from the plastics into the medium upon oocyte maturation was assessed in three independent experiments using 150, 151, and 148 COCs for non-exposed and 148, 150, and 147 COCs for PIC100-exposed media.

In Vitro Fertilization (IVF) and Culture (IVC). Mature COCs were distributed in groups of 35-50 in four-well culture plates (Nunc A/S, Roskilde, Denmark) with $500 \mu \mathrm{L}$ of fertilization medium supplemented with $10 \mu \mathrm{g} / \mathrm{mL}$ heparin, $20 \mu \mathrm{M}$ D-penicillamine, $10 \mu \mathrm{M}$ hypotaurine, and $1 \mu \mathrm{M}$ epinephrine. PS, PDMS, PIC100, E-Shell200, and E-Shell300 polymer structures were added separately to individual fertilization wells; control wells were prepared similarly but without any polymer structure. After co-incubation for $20-22 \mathrm{~h}$ in a humidified atmosphere of $5 \% \mathrm{CO}_{2}$ in air at $38.5{ }^{\circ} \mathrm{C}$, cumulus cells were removed by pipetting, and the presumptive zygotes were cultured in groups of 50 in four-well culture plates with $500 \mu \mathrm{L}$ of synthetic oviductal fluid (SOF) medium, with or without the respective polymer structures. The developing embryos were cultured for 8 days in a humidified atmosphere of $5 \% \mathrm{CO}_{2}$ and $5 \% \mathrm{O}_{2}$ at $38.5{ }^{\circ} \mathrm{C}$. Two days postfertilization (dpf), the number of cleaved embryos was counted after examination using an inverted light microscope with a $20 \times$ objective (Olympus Nederland BV). At eight dpf, the number of expanded blastocysts was determined. Three replicates for control and each of the polymer structure groups were performed (total numbers of COCs used were 604 for control and $380,152,150,150$, and 148 for PIC100, PDMS, PS, EShell200, and E-Shell300 polymers, respectively).

Cell-Based ER-Mediated Bioassay. For the estrogen receptor assay, culture medium with possible leachates from the polymers was used to evaluate any effects on ER activation in the BG1Luc4E2 cell line, as described previously. ${ }^{15}$ This recombinant human ovarian carcinoma (BG-1) cell line is stably transfected with an estrogen receptor element (ERE) and a luciferase transporter gene. ${ }^{16}$ The cells were plated in luminescence 96-well plates at a cell density of $0.4 \times 10^{5}$ cells / well. After $24 \mathrm{~h}$, the cells were exposed to the conditioned media or medium containing estradiol as a positive control. Thereafter, the cells were washed with warm PBS and subsequently lysed with $20 \mu \mathrm{L}$ of lysis buffer (Promega, Leiden, The Netherlands) per well. After $30 \mathrm{~min}, 100 \mu \mathrm{L}$ of a luciferin buffer solution containing $470 \mu \mathrm{M}$ luciferin (Promega) and $530 \mu \mathrm{M}$ ATP (Roche Diagnostics, Leiden, The Netherlands) in MilliQ water at $\mathrm{pH} 7.8$ was added, and luminescence 
A

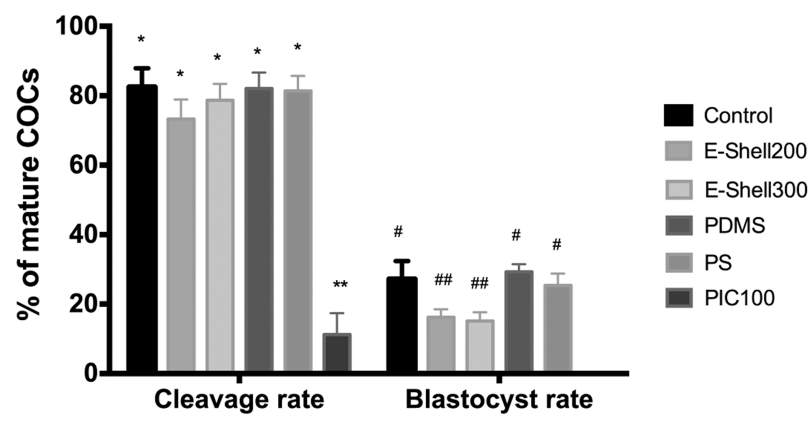

B

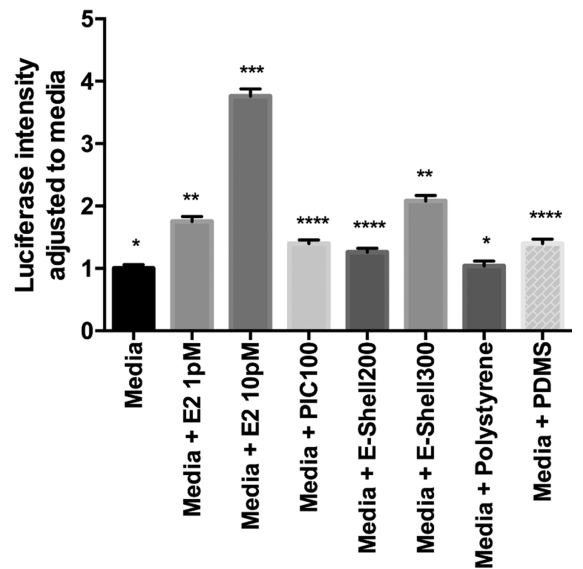

Figure 1. (A) Bovine cleaved embryo and blastocyst production efficiency displayed as percentages of the total number of mature cumulus-oocyte complexes (COCs) submitted for in vitro fertilization (IVF). IVF and in vitro culture (IVC) were performed in the presence of PIC100, E-Shell200, E-Shell300, PDMS, or PS polymer structures. Standard IVF and IVC were also performed without polymer structures (Control). (B) BG1ERE bioassay to detect increases in ER-dependent luciferase activity caused by media conditioned with PIC100, E-Shell-200, E-Shell300, PS, or PDMS, or without any conditioning. An increase in luciferase intensity was found for PIC100, E-Shell-200, E-Shell300, and PDMS; 1 and 10 pM estrogen (E2) were used to check the responsiveness of the cells in the assay. Different asterisks and number signs indicate statistically significant differences $(p<$ $0.05)$.

was measured using a luminometer (Lumistar OPTIMA, BMG Labtech, Ortenberg, Germany).

MS Analysis for Identification of Compounds Leached from the Plastics. A more precise measurement of the components present in the samples exposed to the various polymer materials was performed by direct injection $(5 \mu \mathrm{L} /$ min) of leachates (for leachate isolation, see Supplementary Method 1) into a high-precision/resolution electrospray ionization mass spectrometer (Orbitrap Fusion, Tribrid from Thermo Scientific, Waltham, MA). Electrospray ionization mass spectrometry (ESI-MS) analyses were conducted at 3.5 $\mathrm{kV}$ in positive mode at an ionization temperature of $100{ }^{\circ} \mathrm{C}$ in the $\mathrm{m} / z$ range of $150-4000$, allowing determination of $\mathrm{m} / \mathrm{z}$ values with a precision of 0.001 . For quantification of the peaks at $\mathrm{m} / z 177.06$ and 223.10, diethyl phthalate (Sigma-Aldrich Chemie GmbH, Steinheim, Germany) was first solubilized in a $1: 100(\mathrm{v} / \mathrm{v})$ ratio in distilled ethanol and then further diluted in a concentration series from $10^{-3}$ to $10^{-12} \mathrm{M}$ in Milli- $\mathrm{Q}$ water. This series was injected into the ESI-MS instrument; pure diethyl phthalate (Sigma) was solubilized in 1 volume of ethanol and further diluted with 2 volumes of water to $1 \mu \mathrm{M}$, and concentration was used to check whether this fragmented in a manner similar to that of the $\mathrm{m} / z 223.10$ peak in the polymer-exposed samples (see Figure $S 1$ for the reference mass spectrogram).

Data Analysis. Data were analyzed using IBM SPSS Statistics (version 24). A Shapiro-Wilk test was performed, and all groups were found to be normally distributed. Differences between groups were examined using independent sample $t$ tests $(p<0.05)$.

\section{RESULTS AND DISCUSSION}

The toxicity of polymer structures produced from PIC100, EShell200, E-Shell300, PDMS, and PS and the nature of the toxicants were tested in a biological assay by investigating their effects on early bovine embryo development. Bovine embryo development is a useful and inexpensive model, because it is easy to acquire bovine oocytes (ovaries collected from slaughterhouses) and sperm (from artificial insemination companies) to produce embryos in vitro. Moreover, there is no need for an animal facility, and no ethical restrictions apply to this type of embryo production. As shown in Figure 1A, PIC100 had the strongest negative effect on bovine embryo development, even though it had been subjected to an extensive and stringent washing procedure using solvents (ethanol and isopropanol) and additional light curing before inclusion. Similarly, postcuring methods using extensive solvent washes were previously reported to only marginally improve zebrafish embryo survival when a Visijet Crystal polymer was used to fabricate 3D-printed devices. ${ }^{2}$ The released materials from PIC100 inhibited embryo cleavage across a confluent monolayer culture of oviduct epithelial cells (Figure S2) and also inhibited oocyte maturation (Table SI).

In conflict with the stated biocompatibility according to the international standard ISO 10993, both E-Shell200 and EShell300 had a negative impact on embryo development, although they were less harmful than PIC100 (Figure 1A). Postcuring of E-Shell200 and E-Shell300 did not enhance blastocyst production when compared to no postcuring (16.2 and $15.1 \%$ vs 14.3 and $15.4 \%$, respectively; $p>0.05$ ). Note that the postcuring was performed following the manufacturer's instructions, but using ethanol instead of isopropanol for the sterilization processing right before use. Thus, PIC100, EShell200, and E-Shell300 are not suitable materials for this specific biological use. PDMS was not toxic to (nonbovine) embryos cultured in vitro (Figure 1A), confirming other studies. $^{6,17,18}$ Overall, the unaffected cleavage and blastocyst rates obtained in the presence of both PDMS and PS (PS was obtained commercially as a nontoxic control) illustrate their suitability as materials for manufacturing of advanced in vitro culture platforms and medical devices.

Considering that embryogenesis and cell culture are strongly influenced by the endocrine environment, and that polymers have been shown to leach endocrine-disrupting compounds, ${ }^{19-21}$ we tested whether the polymers compared in this study released components that could activate the ER, 

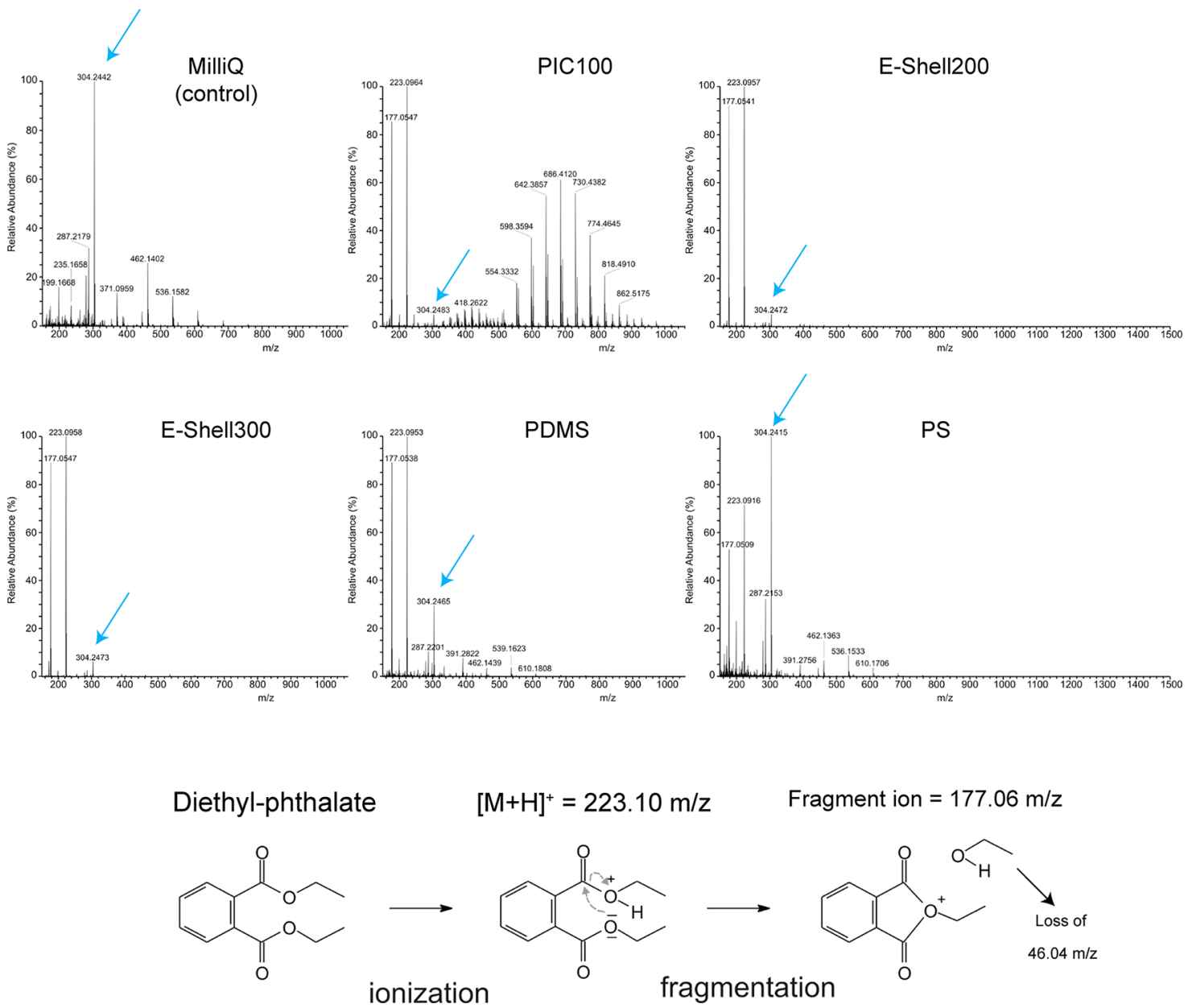

Figure 2. High-resolution ESI-MS analysis of Milli-Q water and Milli-Q water conditioned with PIC100, E-Shell200, E-Shell300, PDMS, or PS. The peaks at $m / z 223.096$ and 177.058 correspond to the positive mother ion and a fragment ion of diethyl phthalate, respectively (see the bottom panel for the formation principle of the fragment ion). The higher-molecular weight peaks $(\mathrm{m} / z 500-1000)$ represent clusters of peaks separated by $m / z$ 44 , which is a characteristic of polyethylene glycol. Blue arrows represent the reference peak at $\mathrm{m} / z 304.24$ found in all samples and used here for the purpose of quantification.

using a cell-based ER reporter gene assay. ${ }^{16,22}$ We observed an increase in the level of transactivation of the ER by all polymers tested, with the exception of PS (Figure 1B). It is worth noting that the ER reporter assay appears to be more sensitive than embryo development given that PDMS activated the ER but did not inhibit blastocyst formation.

We hypothesized that oligomers must leak out of the engineered polymers and contribute to the toxic effects observed. Indeed, leaked compounds could be detected from all five materials, and our results support those of Oskui et al., who detected three different chemical species leaking from stereolithographic 3D-printed devices. ${ }^{1}$

Using matrix-assisted laser desorption/ionization mass spectrometry (MALDI-MS), we detected clusters of peaks separated by $m / z 44$ after they had been conditioned for $24 \mathrm{~h}$ with Milli-Q water with PIC100 and E-Shell200 (Figure S3). The observed spectra correspond to polyethylene glycol (PEG) oligomers. Adverse effects of PEG metabolites on embryo development have been described previously. ${ }^{23,24}$ To further identify low-molecular weight leached components, which could not be detected by MALDI-MS, the samples were also analyzed by high-resolution ESI-MS. The control Milli-Q water contained trace amounts of a compound with an $\mathrm{m} / \mathrm{z}$ value of 304.25 (Figure 2), which corresponds to the molecular formula
$\mathrm{C}_{16} \mathrm{H}_{33} \mathrm{NO}_{4}$ for the non-ionized molecule. This molecule could have originated from any material with which the sample had been in contact (such as Milli-Q anion exchange resins, pipet tips, tubes, and bottles that were used to store or handle the water). Because this peak at $\mathrm{m} / z 304.25$ was present in all spectra, we decided to use it as an internal calibrant to quantify the relative amount of released diethyl phthalate (Table 1). As depicted in Figure 2, two peaks at $\mathrm{m} / z 223.10$ and 177.06 were detected in all water samples exposed to polymer materials. These peaks correspond to a protonated diethyl phthalate compound with a molecular formula of $\mathrm{C}_{12} \mathrm{H}_{24} \mathrm{O}_{4}$ (theoretical $\mathrm{m} / z$ of 223.0965) and a stable fragment of this compound formed by loss of ethanol $\left(\mathrm{C}_{10} \mathrm{H}_{9} \mathrm{O}_{3}\right.$; theoretical $\mathrm{m} / z$ of 177.0546), respectively, for which the fragmentation mechanism is provided in Figure 2. In addition, we injected a sample of $1 \mu \mathrm{M}$ diethyl phthalate into the ESI-MS instrument and found an identical $[\mathrm{M}+\mathrm{H}]^{+}$and a fragment ion peak with an identical fragmentation pattern (see Figure S1). As summarized in Table 1, the relative amounts of diethyl phthalate leached from the polymer materials into the Milli-Q water correlated closely with the level of toxicity detected using the conditioned media for the different polymers.

The concentration of diethyl phthalate released into the water $(78 \mathrm{nM})$ is above the reported reproductive toxic levels 
Table 1. Relative Amounts of Diethyl Phthalate and Its Fragment Ions Present in Milli-Q Samples Conditioned with Different Polymer Materials, Compared to the Control (nonconditioned) Milli-Q Sample

$\begin{array}{lrc}\text { polymer } & \begin{array}{c}\text { diethyl } \begin{array}{c}\text { phthalate ion } \\ \text { events }\end{array} \\ \begin{array}{c}\text { Milli-Q } \\ \text { water }^{a}\end{array}\end{array} & \begin{array}{c}\text { concentration of released diethyl } \\ \text { phthalate }(\mathrm{pM})\end{array} \\ \text { PIC100 } & 0.023 & 0.038 \\ \text { E-Shell200 } & 47109.500 & 78000.00 \\ \text { E-Shell300 } & 381.100 & 630.00 \\ \text { PDMS } & 21.200 & 35.00 \\ \text { PS } & 5.440 & 9.00 \\ & 1.180 & 1.95\end{array}$

${ }^{a}$ Milli-Q water was sham treated in the same way as the Milli-Q water used for plastic conditioning and/or identification of leachates, but without adding a polymer. ${ }^{b}$ Diethyl phthalate ion events $(m / z 223.10$ and 177.06) relative to 100 ion counts of the Milli-Q ion $(m / z$ 304.247).

of phthalates and their metabolites $(10-23 \mathrm{nM}) .^{25,26}$ Phthalates have long been established as endocrine disruptors with toxic effects on mammalian reproduction, and diethyl phthalate was found to be among the most toxic species in exposed rats. ${ }^{27}$ Diethyl phthalate is known as an endocrine disruptor. ${ }^{28-30}$ However, addition of purified diethyl phthalate $(100 \mathrm{nM})$ in IVF and IVC media did not cause any inhibition of blastocyst formation when compared to $0 \mathrm{nM}$ (Figure S4). We consider diethyl phthalate as a marker leachate for plastic toxicity as the nontoxic plastics released only $<1 \mathrm{nM}$ diethyl phthalate.

It is alarming that endocrine disruptors were released from all polymers. PIC100, E-Shell200, and E-Shell300 are polymers that are available commercially for stereolithography-based 3D printing. PIC100 is mostly used to prototype jewelry and tool models but has also been used to develop cell culture devices. $^{31,32}$ E-Shell200 and E-Shell300 are stated to be biocompatible and mostly used to prototype hearing aid products, otoplastics, and medical devices. However, little is known about the effects of long-term exposure to these materials on personnel involved in the production of these polymers or patients that carry them. PDMS is widely used for microfluidic device fabrication, ${ }^{33}$ and PS is the main plastic used to make culture dishes. Although the level of release of phthalates from PDMS was low, it is worth considering the possibility of adverse effects after long-term continuous exposure. It is important to consider that an increasing amount of 3D-engineered polymers and waste from printing or molding with these polymers is being introduced into the environment. We should prioritize strategies for safe collection and disposal of polymers and waste materials generated by $3 \mathrm{D}$ printing and molding and monitor the effects of contact with the polymers. Particular attention should be paid to commercial materials that can be used in household 3D printers. Domestic consumers are generally less aware of the need for proper disposal and handling of toxic polymers and may, unintentionally, contribute to the release of endocrine disruptors (such as phthalates) into the environment.

\section{ASSOCIATED CONTENT}

\section{(S) Supporting Information}

The Supporting Information is available free of charge on the ACS Publications website at DOI: 10.1021/acs.estlett.7b00495.
Collection of leachates from plastics for toxicological assays, ESI-MS output of $1 \mu \mathrm{M}$ diethyl phthalate, effects of PIC100 leachates on oocyte maturation, in vitro fertilization and culture using 3D-cultured bovine oviduct epithelial cells, MALDI analysis methods and output, and results of DEP on embryo cleavage and blastocyst rate (PDF)

\section{AUTHOR INFORMATION}

\section{Corresponding Author}

*E-mail: b.m.gadella@uu.nl. Phone: +31302535386.

ORCID (0)

Barend M. Gadella: 0000-0001-9618-1261

Notes

The authors declare no competing financial interest.

\section{REFERENCES}

(1) Oskui, S. M.; Diamante, G.; Liao, C.; Shi, W.; Gan, J.; Schlenk, D.; Grover, W. H. Assessing and reducing the toxicity of 3D-printed parts. Environ. Sci. Technol. Lett. 2016, 3 (1), 1-6.

(2) Macdonald, N. P.; Zhu, F.; Hall, C. J.; Reboud, J.; Crosier, P. S.; Patton, E. E.; Wlodkowic, D.; Cooper, J. M. Assessment of biocompatibility of 3D printed photopolymers using zebrafish embryo toxicity assays. Lab Chip 2016, 16 (2), 291-297.

(3) Popov, V. K.; Evseev, A. V.; Ivanov, A. L.; Roginski, V. V.; Volozhin, A. I.; Howdle, S. M. Laser stereolithography and supercritical fluid processing for custom-designed implant fabrication. J. Mater. Sci.: Mater. Med. 2004, 15 (2), 123-128.

(4) Inoue, Y.; Ikuta, K. Detoxification of the photocurable polymer by heat treatment for microstereolithography. Procedia CIRP 2013, 5, $115-118$.

(5) Stephens, B.; Azimi, P.; El Orch, Z.; Ramos, T. Ultrafine particle emissions from desktop 3D printers. Atmos. Environ. 2013, 79, 334339.

(6) Esteves, T. C.; van Rossem, F.; Nordhoff, V.; Schlatt, S.; Boiani, M.; Le Gac, S. A microfluidic system supports single mouse embryo culture leading to full-term development. RSC Adv. 2013, 3 (48), 26451.

(7) Huh, D.; Kim, H. J.; Fraser, J. P.; Shea, D. E.; Khan, M.; Bahinski, A.; Hamilton, G. a.; Ingber, D. E. Microfabrication of human organson-chips. Nat. Protoc. 2013, 8 (11), 2135-2157.

(8) Wolbers, F.; ter Braak, P.; Le Gac, S.; Luttge, R.; Andersson, H.; Vermes, I.; van den Berg, A. Viability study of HL60 cells in contact with commonly used microchip materials. Electrophoresis 2006, 27 (24), 5073-5080.

(9) Colborn, T.; vom Saal, F. S.; Soto, A. M. Developmental effects of endocrine-disrupting chemicals in wildlife and humans. Environ. Health Perspect. 1993, 101 (5), 378-384.

(10) Chen, X.; Xu, S. S. J. L.; Tan, T.; Lee, S. T.; Cheng, S. H.; Lee, F. W. F.; Xu, S. S. J. L.; Ho, K. C. Toxicity and estrogenic endocrine disrupting activity of phthalates and their mixtures. Int. J. Environ. Res. Public Health 2014, 11 (3), 3156-3168.

(11) Rattan, S.; Zhou, C.; Chiang, C.; Mahalingam, S.; Brehm, E.; Flaws, J. A. Exposure to endocrine disruptors during adulthood: consequences for female fertility. J. Endocrinol. 2017, 233 (3), R109R129.

(12) Kajta, M.; Wójtowicz, A. K. Impact of endocrine-disrupting chemicals on neural development and the onset of neurological disorders. Pharmacol. Rep. 2013, 65 (6), 1632-1639.

(13) Kuo, C.-H.; Yang, S.-N.; Kuo, P.-L.; Hung, C.-H. Immunomodulatory effects of environmental endocrine disrupting chemicals. Kaohsiung J. Med. Sci. 2012, 28 (Suppl. 7), S37-542.

(14) Van Tol, H. T. A. A.; Van Eerdenburg, F. J. C. M. C. M.; Colenbrander, B.; Roelen, B. A. J. J. Enhancement of bovine oocyte maturation by leptin is accompanied by an upregulation in mRNA 
expression of leptin receptor isoforms in cumulus cells. Mol. Reprod. Dev. 2008, 75 (4), 578-587.

(15) van Duursen, M. B. M.; Smeets, E. E. J. W.; Rijk, J. C. W.; Nijmeijer, S. M.; van den Berg, M. Phytoestrogens in menopausal supplements induce ER-dependent cell proliferation and overcome breast cancer treatment in an in vitro breast cancer model. Toxicol. Appl. Pharmacol. 2013, 269 (2), 132-140.

(16) Rogers, J. M.; Denison, M. S. Recombinant cell bioassays for endocrine disruptors: development of a stably transfected human ovarian cell line for the detection of estrogenic and anti-estrogenic chemicals. In Vitro Mol. Toxicol. 2000, 13 (1), 67-82.

(17) Raty, S.; Walters, E. M.; Davis, J.; Zeringue, H.; Beebe, D. J.; Rodriguez-Zas, S. L.; Wheeler, M. B. Embryonic development in the mouse is enhanced via microchannel culture. Lab Chip 2004, 4 (3), 186.

(18) Kieslinger, D. C.; Hao, Z.; Vergouw, C. G.; Kostelijk, E. H.; Lambalk, C. B.; Le Gac, S. In vitro development of donated frozenthawed human embryos in a prototype static microfluidic device: a randomized controlled trial. Fertil. Steril. 2015, 103 (3), 680-686.e2.

(19) Choi, B.-I.; Harvey, A. J.; Green, M. P. Bisphenol A affects early bovine embryo development and metabolism that is negated by an oestrogen receptor inhibitor. Sci. Rep. 2016, 6 (1), 29318.

(20) Pocar, P.; Perazzoli, F.; Luciano, A. M.; Gandolfi, F. In vitro reproductive toxicity of polychlorinated biphenyls: Effects on oocyte maturation and developmental competence in cattle. Mol. Reprod. Dev. 2001, 58 (4), 411-416.

(21) Sone, K.; Hinago, M.; Kitayama, A.; Morokuma, J.; Ueno, N.; Watanabe, H.; Iguchi, T. Effects of $17 \beta$-estradiol, nonylphenol, and bisphenol-A on developing Xenopus laevis embryos. Gen. Comp. Endocrinol. 2004, 138 (3), 228-236.

(22) Ahn, K. C.; Zhao, B.; Chen, J.; Cherednichenko, G.; Sanmarti, E.; Denison, M. S.; Lasley, B.; Pessah, I. N.; Kültz, D.; Chang, D. P. Y.; et al. In vitro biologic activities of the antimicrobials triclocarban, its analogs, and triclosan in bioassay screens: receptor-based bioassay screens. Environ. Health Perspect. 2008, 116 (9), 1203-1210.

(23) Klug, S.; Merker, H. J.; Jäckh, R. Effects of ethylene glycol and metabolites on in vitro development of rat embryos during organogenesis. Toxicol. In Vitro 2001, 15 (6), 635-642.

(24) Carney, E. W.; Tornesi, B.; Markham, D. A.; Rasoulpour, R. J.; Moore, N. Species-specificity of ethylene glycol-induced developmental toxicity: toxicokinetic and whole embryo culture studies in the rabbit. Birth Defects Res., Part B 2008, 83 (6), 573-581.

(25) Du, Y.-Y.; Fang, Y.-L.; Wang, Y.-X.; Zeng, Q.; Guo, N.; Zhao, H.; Li, Y.-F. Follicular fluid and urinary concentrations of phthalate metabolites among infertile women and associations with in vitro fertilization parameters. Reprod. Toxicol. 2016, 61, 142-150.

(26) Kalo, D.; Roth, Z. Low level of mono(2-ethylhexyl) phthalate reduces oocyte developmental competence in association with impaired gene expression. Toxicology 2017, 377, 38-48.

(27) Singh, A. R.; Lawrence, W. H.; Autian, J. Teratogenicity of phthalate esters in rats. J. Pharm. Sci. 1972, 61 (1), 51-55.

(28) Kumar, N.; Sharan, S.; Srivastava, S.; Roy, P. Assessment of estrogenic potential of diethyl phthalate in female reproductive system involving both genomic and non-genomic actions. Reprod. Toxicol. 2014, 49, 12-26.

(29) Parveen, M.; Inoue, A.; Ise, R.; Tanji, M.; Kiyama, R. Evaluation of estrogenic activity of phthalate esters by gene expression profiling using a focused microarray (EstrArray). Environ. Toxicol. Chem. 2008, 27 (6), 1416-1425.

(30) Christen, V.; Crettaz, P.; Oberli-Schrämmli, A.; Fent, K. Some flame retardants and the antimicrobials triclosan and triclocarban enhance the androgenic activity in vitro. Chemosphere 2010, 81 (10), $1245-1252$.

(31) Ferraz, M. A. M. M.; Henning, H. H. W.; Costa, P.; Jos, M.; Melchels, F. P. W.; Wubbolts, R.; Stout, T. A. E.; Vos, P. L. A. M.; Gadella, B. M. Improved bovine embryo production in an oviduct-ona-chip system: Prevention of poly-spermic fertilization and parthenogenic activation. Lab Chip 2017, 17, 905-916.
(32) Costa, P. F.; Albers, H. J.; Linssen, J. E. A.; Middelkamp, H. H. T.; van der Hout, L.; Passier, R.; van den Berg, A.; Malda, J.; van der Meer, A. D. Mimicking arterial thrombosis in a 3D-printed microfluidic in vitro vascular model based on computed tomography angiography data. Lab Chip 2017, 17 (16), 2785-2792.

(33) Duffy, D. C.; McDonald, J. C.; Schueller, O. J. A.; Whitesides, G. M. Rapid prototyping of microfluidic systems in poly(dimethylsiloxane). Anal. Chem. 1998, 70 (23), 4974-4984. 